\title{
Correspondence
}

\section{Letter to the Editor and reply concerning: Hip development after surgery to prevent hip dislocation in cerebral palsy: a longitudinal register study of 252 children}

Acta Orthop 2022; 93: 45-50. DOI 10.1080/17453674.2021.1989563

Sir,-The Swedish surveillance program for children with CP (CPUP, Cerebral Palsy Follow-up Program) was initiated in 1994 and consists of multidisciplinary, long-term follow-up of joint motion and musculoskeletal function in order to enable early intervention to prevent permanent disability. Recently, Acta Orthopaedica published a longitudinal register study based on hip surveillance in CPUP (Wagner and Hägglund 2022). The study analysed the postoperative development of MP (migration percentage) after surgery to prevent hip dislocation. This is a clinically important question since the outcome of such surgery has been the subject of controversy for many years. 252 children at GMFCS levels III-V were studied. The surgical methods included adductor-psoas lengthening (APL) or varus derotation femoral osteotomy (VDRO). After a follow-up of $\geq 3$ years, 54 of the 158 patients (34\%) with APL needed reoperation, whereas 15 of 94 patients with VDRO were reoperated (16\%).

Some methodological weaknesses reduced the value of the study. First, MP and reoperation were the only outcome measures. The study would be more clinically interesting and easier to compare with previous studies if the failure rate, defined as the sum of reoperations and cases with MP $\geq 50 \%$ in accordance with previous studies (Shore et al. 2015, Terjesen 2019), had been used instead of reoperations only. Secondly, the follow-up period was rather short, since there is a deterioration of outcome with time. Shore et al. (2015) reported failure rates of $24 \%$ after 5 years and $42 \%$ after 10 years, and Terjesen (2019) found a mean time to failure of 3.6 years after femoral and pelvic osteotomies. Thus, the rate of reoperations was probably underestimated in the present study and there was no Kaplan-Meier survival analysis with time to reoperation as endpoint. In a similar study, which was also based on CPUP data (Kiapekos et al. 2019), the study design was satisfactory and included follow-up of $\geq 5$ years, failure rates according to Shore et al. (2015), and survival analysis. The present study did not compare the results with those of Kiapekos et al. (2019). Thus, there was no discussion of the marked difference in the reoperation rates after VDRO $(16 \%$ in the present study and $39 \%$ in the previous one). Possible reasons for this discrepancy should be discussed, especially since both studies were based on CPUP data and many patients probably were participants in both studies.
In spite of these objections, further studies based on the CPUP and similar registers should be encouraged. The advantages are obvious: large numbers of patients can be included that make it possible to analyse subgroups like GMFCS levels and age groups, and systematic surveillance programs are suitable for longitudinal studies. Certain requirements for future studies should be defined. The years of birth and the years of treatment should be specified, as should gender, age and GMFCS levels. Pre- and postoperative pain and quality of life should be analysed, using standardized classifications. Postoperative follow-up should be satisfactory, i.e. $\geq 5$ years. Classification of outcome should be in accordance with internationally accepted gradings in order to compare the results with those of previous studies. Last, but not least, results of previous studies based on the same surveillance register should be accounted for and discussed to detect possible changes over time.

\section{Terje Terjesen \\ Division of Orthopaedic Surgery, Oslo University Hospital, Norway \\ e-mail:terje.terjesen@rikshospitalet.no}

Sir,-Terje Terjesen has misunderstood the purpose of our study and that explains the objections he raises.

A study must be designed to address the research question. The purpose of our study was to analyze the pattern of radiographic development of Migration Percentage (MP) before and after surgery with adductor psoas lengthening and varus derotation osteotomy to prevent hip dislocation in cerebral palsy.

If our purpose had been to study clinical outcomes after surgery, we would certainly have chosen a longer postoperative follow-up period. Furthermore, we would clearly have referred to previous studies with the same purpose, and we would have chosen appropriate outcome measures such as acetabular dysplasia, femoral head morphology, range of motion, pain, and patient satisfaction. However, that was not the purpose of the current study and, consequently, it was not done. 
Finally, we have clearly stated that there are probably hips that are reoperated after the census date and discussed the consequences this might have for the results in the Discussion (paragraph 5).

\section{Philippe Wagner and Gunnar Hägglund} e-mail: philippe.wagner@regionvastmanland.se
Kiapekos N, Broström E, Hägglund G, Åstrand P. Primary surgery to prevent hip dislocation in children with cerebral palsy in Sweden: a minimum 5-year follow-up by the national surveillance program (CPUP). Acta Orthop 2019; 90: 495-500.

Shore BJ, Zurakowski D, Dufreny C, Powell D, Matheney T H, Snyder B D. Proximal femoral varus derotation osteotomy in children with cerebral palsy: the effect of age, gross motor function classification system level, and surgeon volume on surgical success. J Bone Joint Surg Am 2015; 97 : 2024-31.

Terjesen T. Femoral and pelvic osteotomies for severe hip displacement in nonambulatory children with cerebral palsy: a prospective populationbased study of 31 patients with 7 years follow-up. Acta Orthop 2019; 90: 614-21.

Wagner P, Hägglund G. Hip development after surgery to prevent hip dislocation in cerebral palsy: a longitudinal register study of 252 children. Acta Orthop 2022; 93: 45-50. 